\title{
«Huitante» ou «quatre-vingts»? (Im)mobilité et stratification sociale à l'université
}

Cédric Margot et Gilles Merminod

\section{(2) OpenEdition}

\section{Journals}

Édition électronique

URL : http://journals.openedition.org/edl/2316

DOI : $10.4000 /$ edl.2316

ISSN : 2296-5084

\section{Éditeur}

Université de Lausanne

\section{Édition imprimée}

Date de publication : 15 mars 2020

Pagination : 15-20

ISBN : 978-2-940331-73-4

ISSN : 0014-2026

\section{Référence électronique}

Cédric Margot et Gilles Merminod, « «Huitante» ou «quatre-vingts»? (Im)mobilité et stratification sociale à l'université », Études de lettres [En ligne], 312 | 2020, mis en ligne le 24 mars 2020, consulté le 17 septembre 2020. URL : http://journals.openedition.org/edl/2316 ; DOI : https://doi.org/10.4000/edl 2316

\section{(C) Études de lettres}




\section{"HUITANTE» OU «QUATRE-VINGTS"? (IM)MOBILITÉ ET STRATIFICATION SOCIALE À L'UNIVERSITÉ}

Si plus d'une cinquantaine d'enseignants chercheurs de la Faculté des lettres de l'Université de Lausanne ont écrit sur 1980 dans ce volume, on ne sait pas grand-chose sur la façon dont ils pratiquent ce nombre dans leur usage quotidien du langage: disent-ils «mille neuf cent huitante» ou "mille neuf cent quatre-vingts»? La question pourrait paraître futile, à peine digne d'attention. Et pourtant, chercher à y répondre nous informe des réalités diverses vécues par les membres d'une institution, la Faculté des lettres, révélatrice d'une société contemporaine où celles et ceux en position de domination culturelle, économique et sociale sont mobiles et présentent des pratiques langagières "fluides", alors que les autres paraissent s'inscrire dans un rapport plus affirmé aux normes locales.

Dans la population vaudoise, il existe un sentiment de subordination à l'égard du français parlé en France, qui s’articule à une forme de dépréciation de la variété locale. L'emploi de la variante "huitante» - pratique majoritaire dans les cantons de Vaud et de Fribourg et encore largement répandue en Valais - fait néanmoins exception: les locuteurs «invoquant l'histoire, la logique et le génie de la langue [pour] la légitimer [...] aidés par une certaine pratique "officielle" de la langue locale qui milite par exemple, que ce soit à l'école ou dans les médias, en faveur du recours aux formes archaïques des nombres» ${ }^{1}$. L'emploi ponctuel de "quatre-vingts» est, quant à lui, associé à un effort pour garantir l'intercompréhension

I. P. Singy (éd.), Identités de genre, identités de classe et insécurité linguistique, p. 129. 
entre francophones provenant de régions différentes. L’emploi majoritaire de «huitante» se vérifie-t-il en Faculté des lettres? Et y associe-t-on les mêmes raisons d'emploi?

Pour investiguer ce phénomène, nous avons envoyé un questionnaire à l'ensemble des membres de la Faculté des lettres, ceci englobant les corps enseignant, étudiant, administratif et technique. Ce questionnaire, conçu dans la tradition sociolinguistique et composé d'une quinzaine de questions, visait à documenter les pratiques déclarées par les membres de la Faculté des lettres et les représentations y étant associées, ainsi que certaines caractéristiques sociales et biographiques des personnes interrogées. Ceci nous a permis d'observer qui utilise quelle(s) variante(s), avec qui, quand et pourquoi. Sur l'ensemble des membres de la Faculté des lettres (environ 2750 personnes), nous avons obtenu 376 réponses complètes. En synthèse, notre échantillon de réponses reflète raisonnablement les caractéristiques de la population interrogée et présente la structuration suivante: sur le plan du genre, 230 femmes, 126 hommes, et 20 personnes ne souhaitant pas déclarer de genre; sur le plan socioprofessionnel, 259 membres du corps étudiant, 120 membres du personnel d'enseignement et de recherche, et 19 membres du personnel administratif et technique (il était possible de figurer dans plusieurs catégories); sur le plan des catégories d'âge représentées, 245 personnes ayant moins de trente ans, 61 entre 31 et 40 ans, 38 entre 41 et 50 ans, et 32 de plus de 50 ans.

La communauté investiguée présente une stratification de par la fonction qu'occupent ses membres (étudiants, personnel d'enseignement et de recherche, et personnel administratif et technique), cette stratification allant de pair avec une gradation de l'âge (les étudiants ayant pour la plupart moins de 30 ans) et une hétérogénéité croissante du lieu de scolarisation primaire (78\% des étudiants ont été scolarisés dans les cantons de Vaud ou du Valais, alors que ce n'est le cas que pour $46,7 \%$ du personnel d'enseignement et de recherche). Ainsi, si l'on croise les déclarations que font les membres de la Faculté des lettres à propos de leur usage de "huitante» ou de "quatre-vingts" avec l'âge qu'ils indiquent avoir, on observe que les personnes de moins de 30 ans disent dans la très grande majorité n'utiliser que «huitante», alors que cet usage exclusif va décroissant quand l'âge des participants augmente. Sachant que la plupart des personnes de moins de 30 ans sont des étudiants dont le lieu de scolarisation primaire est le canton de Vaud ou le canton du 
Valais, la seule connaissance du lieu de scolarisation primaire est, en toute logique, un indicateur de la pratique déclarée par le locuteur ou la locutrice. Néanmoins, plus l'âge avance, plus on remarque la prévalence d'un usage non exclusif d'une variante: par exemple, $71 \%$ des étudiants ayant été scolarisés en primaire dans les cantons de Vaud ou du Valais disent utiliser exclusivement "huitante», alors que ce pourcentage tombe à $41 \%$ pour la population des enseignants-chercheurs ayant le même profil de scolarisation $(\mathrm{P}=0.000)$. On pourrait expliquer cette tendance de la manière suivante: la fonction d'enseignant-chercheur mène à des contacts plus fréquents avec des locuteurs et des locutrices dont l'usage diffère de celui attesté majoritairement dans le canton de Vaud. Il faut enfin observer que sur l'ensemble de l'échantillon le genre est une variable déterminante $(\mathrm{P}=0.038)$; les femmes présentant majoritairement une pratique exclusive de «huitante». Cette donnée s'explique par l'importante présence des femmes dans la population étudiante (en comparaison du groupe des enseignants-chercheurs), population elle-même majoritairement issue des systèmes scolaires vaudois et valaisans.

La première raison donnée par les participants pour expliquer leur usage principal de "huitante» ou de "quatre-vingts" est celle de la socialisation langagière primaire, c'est-à-dire le fait d'avoir acquis l'une ou l'autre variante en premier («Parce que c'est comme ça que je l'ai appris, c'est comme ça qu'on le dit chez moi»), l'emploi étant alors présenté comme une forme d'automatisme ("Sans raison, c'est un réflexe»). Conjointement, l'usage ponctuel de l'une ou l'autre variante est souvent associé à une situation d'ajustement à l’auditoire («Principalement lorsque je dois m'adapter à une personne qui ne connaît pas huitante"; "Je suis Français. Je n'utilise huitante qu'en Suisse ou en Belgique. Je considère l'emploi d'un usage local comme une marque de politesse et d'intégration»). On note par ailleurs des raisons spécifiques justifiant l'emploi de «huitante». D'une part, il y a celle de la mise en valeur de l'appartenance régionale et de son patrimoine linguistique ("parce que je suis une bonne Vaudoise»; "Pour utiliser une variante de langage typiquement romande et maintenir cette spécificité vivante»). D'autre part, certains évoquent l'ergonomie étymo-logique de l'expression «huitante", perçue à la fois comme plus systématique du point de vue morphologique («il est, selon moi, plus logique de dire huitante dans la continuité de soixante, septante, etc. au regard des chiffres six, sept, huit, neuf"; "parce que sept $\rightarrow$ SEPTante alors huit $\rightarrow$ HUITante») et plus facile d'emploi ("Je 
suis d'origine des Pays-Bas, à l'école, j'ai donc appris l'usage de quatrevingts, mais je me sens beaucoup plus à l'aise avec huitante et j'ai décidé de rester avec»). Enfin, on remarque également l'emploi de "huitante" à des fins d'inclusion sociale ("J'habite Lausanne depuis $\mathrm{N}$ ans et je pense [...] que dire "huitante" est un facteur d'intégration dans cette ville qui se construit dans une sorte de compétition avec sa voisine du bout du lac et où, de façon générale, il n'est pas très facile de se faire un cercle d'amis»). Quant aux raisons données spécifiquement pour l'emploi de "quatre-vingts", certains participants font référence au capital symbolique ("C'est plus chic») ou esthétique («Parce que cela sonne mieux et à l'oreille me semble plus agréable») véhiculés par ces usages, signalant que cette variante est considérée comme la norme de prestige. En écho à cette dimension, certaines personnes déclarent utiliser "quatre-vingts " à des fins ludiques, dans une logique de renversement des codes ("[...] parfois par snobisme de langage, parfois par blague»; "par amour de l'absurde et du byzantinisme»).

Revenons maintenant à 1980. Dit-on "mille neuf cent huitante» ou «mille neuf cent quatre-vingts» en Faculté des lettres? Hormis quelques exceptions (notamment la collocation "années quatre-vingt»: "Quand je veux parler des années, ou de la musique de telles années, j'emploie quatre-vingts»), la réponse est comparable aux analyses que nous avons proposées ci-devant pour les expressions "huitante» et "quatre-vingts", pour autant que les participants aient une pratique en phase avec ce qu'ils déclarent.

Cédric Margot

Gilles Merminod

Section des sciences du langage et de l'information, Faculté des lettres, Université de Lausanne 


\section{BIBLIOGRAPHIE}

Avanzi, Mathieu, Atlas du français de nos régions, Paris, Armand Colin, 2017.

Blommaert, Jan, The Sociolinguistics of Globalization, Cambridge, Cambridge University Press, 2010.

Coupland, Nikolas (éd.), The Handbook of Language and Globalization, Chichester, Wiley Blackwell, 2010.

Meyerhoff, Miriam, Schleef, Erik, MacKenzie, Laurel, Doing Sociolinguistics. A Practical Guide to Data Collection and Analysis, London, Routledge, 2015.

Schleef, Erik, "Written Surveys and Questionnaires in Sociolinguistics", in Research methods in sociolinguistics, ed. by Janet Holmes, Kirk Hazen, Oxford, Wiley Blackwell, 2013, p. 42-57.

Singy, Pascal, L'image du français en Suisse romande: une enquête sociolinguistique en Pays de Vaud, Paris, L'Harmattan, 1996.

- (éd.), Identités de genre, identités de classe et insécurité linguistique, Berne, Peter Lang, 2004.

Thurlow, Crispin, Jaworski, Adam, "The discursive production and maintenance of class privilege: Permeable geographies, slippery rhetorics", Discourse \& Society, 28 (2017), p. 535-558. 
